\title{
The CoRoT Exoplanet program: status \& results
}

\author{
M. Deleuil ${ }^{1}$, C. Moutou ${ }^{1}$, P. Bordé ${ }^{2} \&$ the CoRoT exoplanet science team \\ ${ }^{1}$ Laboratoire d'Astrophysique de Marseille, 38 rue F. Joliot-Curie, 13388 \\ Marseille Cedex 13, France
}

${ }^{2}$ Institut d'Astrophysique Spatiale, Bât. 121, 91405 Orsay, France

\begin{abstract}
The CoRoT satellite is the first instrument hunting for planets from space. We will review the status of the CoRoT/Exoplanet program. We will then present the CoRoT exoplanetary systems and how they widen the range of properties of the close-in population and contribute to our understanding of the properties of planets.
\end{abstract}

\section{Introduction}

CoRo $T^{1}$ has been designed with two distinct scientific objectives which both require continuous observations and ultra-high precision relative stellar photometry: one is the detection of extrasolar planets by the transit method; the second is the study of stellar interiors by asteroseismology (Baglin et al. 2009). In addition, the long duration of the observation periods together with the exquisite photometric precision allow a number of stellar physics studies. The instrument started the scientific observations on February 2nd 2007. The mission was initially scheduled for 3 years but extended for 3 additional years, that is till March 2013. Auvergne et al. (2008) give a complete description of the instrument in flight based on raw and calibrated data. In the present paper, we will just recall the main characteristics of the mission and outline some of its results.

\section{CoRoT, brief summary}

\subsection{Photometry}

Typical exoplanet targets have a $\mathrm{V}$-magnitude in the range $\simeq 11$ up to 16 . The photometry is performed on board for up to 6000 selected targets per CCD, 2 CCDs being dedicated to the exoplanet program. For a given target, the flux is thus integrated over a pre-designed photometric mask that encompasses the quite large PSF of the exoplanet channel, whose typical size is about 35 " x 23 ". For stars brighter than magnitude 15, the pixels within the photometric mask are separated in 3 sub-sets according to the percentage of stellar flux. The co-addition of the pixels intensity over these 3 areas

\footnotetext{
${ }^{1}$ The CoRoT space mission has been developed and is operated by CNES, with contributions from Austria, Belgium, Brazil, ESA, Germany and Spain.
} 
Table 1: CoRoT runs from 2007 to 2010.

\begin{tabular}{lccrrrr}
\hline Run & $\begin{array}{c}\text { Date } \\
\text { start }\end{array}$ & $\begin{array}{c}\text { Duration } \\
{[\mathrm{d}]}\end{array}$ & $\begin{array}{r}\text { Nb of } \\
\text { Targets }\end{array}$ & $\begin{array}{r}\text { Nb } \\
\text { Cand. }\end{array}$ & $\begin{array}{r}\text { to } \\
\text { FUp }\end{array}$ & $\begin{array}{c}\text { Data } \\
\text { Status }\end{array}$ \\
\hline IRa01 & $02 / 2007$ & 45 & 9921 & 254 & 40 & Public \\
SRc01 & $04 / 2007$ & 26 & 7015 & 261 & 62 & Public \\
$L R c 01$ & $05 / 2007$ & 152 & 11448 & 229 & 29 & Public \\
$L R a 01$ & $10 / 2007$ & 150 & 11448 & 304 & 79 & Public \\
SRa01 & $03 / 2008$ & 25 & 8189 & 163 & 29 & Public \\
$L R c 02$ & $04 / 2008$ & 150 & 11448 & 286 & 56 & Public \\
$S R c 02$ & $09 / 2008$ & 21 & 11448 & 336 & 45 & Public \\
SRa02 & $10 / 2008$ & 32 & 10305 & 217 & 32 & Public \\
$L R a 02$ & $11 / 2008$ & 115 & 11448 & 362 & 36 & Public \\
$L R c 03$ & $04 / 2009$ & 89 & 5724 & 244 & 61 & Public \\
$L R c 04$ & $07 / 2009$ & 83 & 5724 & 173 & 51 & CoIs \\
$L R a 03$ & $10 / 2009$ & 148 & 5329 & 124 & 22 & CoIs \\
SRa03 & $03 / 2010$ & 24 & 4169 & 100 & 15 & on alarm \\
$L R c 05$ & $04 / 2010$ & 84 & 5724 & 89 & 22 & on alarm \\
$L R c 06$ & $07 / 2010$ & 77 & 5724 & 100 & 21 & on alarm \\
\hline
\end{tabular}

Column 5: number of transit candidates detected; Column 6: number of candidates assessed worthy of follow-up observations. Following the DPU 1 break down in March 2009, the number of light curves is divided per 2 in the last fields.

provides 3 different light curves, referred to as the red, the green and the blue light curves. These light curves have however no direct correspondence to any standard photometric system. For fainter stars, only the standard single-band photometry (white) is performed. The integration time on board is $32 \mathrm{sec}$ but the flux of 16 read-outs is coadded on board over an $8.5 \mathrm{~min}$ time span before being downloaded. The elementary $32 \mathrm{sec}$ integration time is however preserved for selected targets, whose list is regularly updated during CoRoT observations thanks to the Alarm mode (Surace et al. 2008). In march 2009, one of the two DPUs which pilot the on-board photometric treatment broke down and could not be switched back on to operation. As a consequence, the field-of-view for exoplanet search is reduced to $\left(1.35^{\circ}\right)^{2}$.

\subsection{Observation strategy}

The instrument has 2 continuous viewing zones which are two circles of $\simeq 10^{\circ}$ of radius, centered in the galactic plane and separated by $180^{\circ}$ in right ascension. This ensures a level of background light due to the Earth reflected light as low as possible. In addition, in order to avoid to be blinded by the Sun, twice a year the instrument is rotated and is pointed in the opposite direction. The strategy of observation is flexible but consists mainly in one long run (LR) lasting for about 140 days and one shorter run (SR) whose duration is between 20 and 30 days per half-year. The fields observed in the circle centered at $6^{h} 50^{\mathrm{m}}$ in right ascension are referred as "anti-center fields", and those in the circle at $18^{h} 50^{m}$, as "center fields". Table 1 gives a summary of the stellar fields that have been observed since the beginning of the operations. With only one operational CCD left, the strategy of observation has been adapted and some of the long runs have been replaced by two runs of a shorter duration.

Figure 1 shows the location of the various CoRoT exoplanet fields inside the two CoRoT 

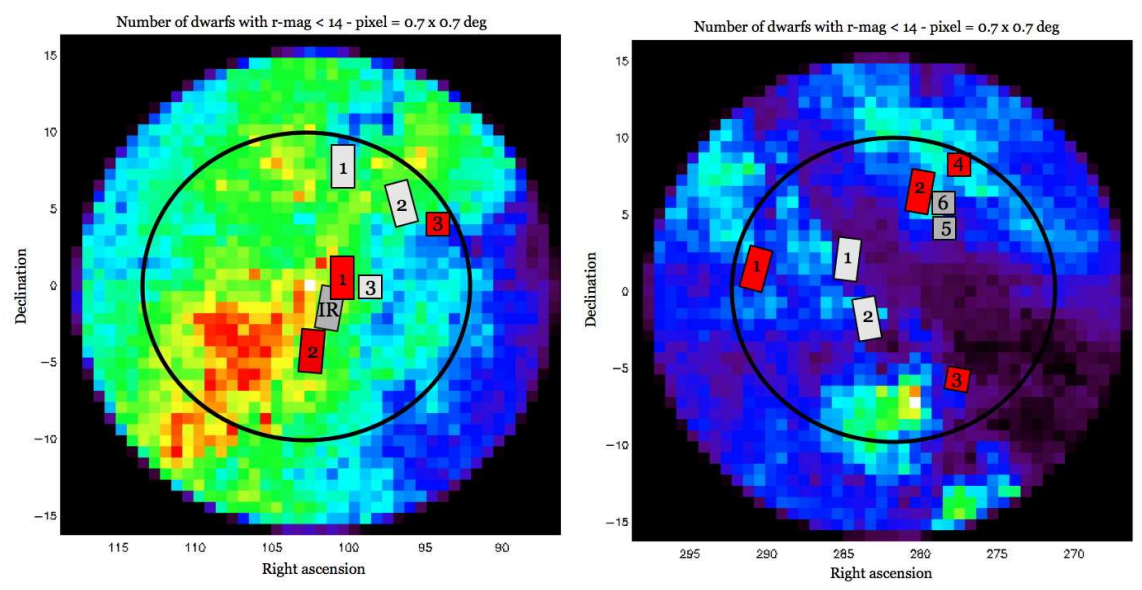

Figure 1: The two continuous pointing zones of CoRoT. The small rectangular and square shapes show the exoplanet fields that have been observed up to summer 2010. The color scale indicate the density of dwarf stars with $r$-mag $<14$. Note the extreme reddening variations towards the center fields.

continuous viewing zones overplotted on a dwarf density map. The two regions have different stellar content in terms of dwarf and giant but also in their distribution over the various spectral type (Gazzano et al. 2010). This allows to probe regions in the galactic plane with different stellar properties in terms of metallicity but also in terms of age. Once the statistics of planets found by CoRoT becomes much more significant, this should bring some insights into the physical conditions that prevail for planet formation mechanisms.

\section{Detection capacity}

In summer 2010, a total of 129326 light curves has been collected (Table 1). These light curves show a fascinating diversity with various kind of stellar behavior: quiet stars display nearly constant a flux while others exhibit a strong variability over a time scale of a few minutes (Fig. 2). All these light curves are nevertheless analyzed for transit searches. Transits are detected in about 100 up to 300 light curves per run, that is, in about 3000 light curves for the whole mission to date. They present various depths, shapes and durations. These detected transits are however predominantly transiting stellar systems. About $80 \%$ to $90 \%$ of these stellar systems are pinpointed thanks to the long duration of the light curves, through the identification of the secondary transits or light curve modulation. The remaining candidates are screened out by follow-up observations, using various techniques from photometric observations to radial velocity measurements. These follow-up observations play a major role in the CoRoT science and require a huge observational effort. They allow to assess the nature of the detected transiting body, stellar or planetary and, in the later case, to measure its mass. Bouchy et al. (2009) give a complete overview of the strategy for radial velocity observations and Deeg et al. (2009) present the ground-based photometric follow-up one.

Whatever their nature, stellar or planetary, one can use the detected transits to assess the performance of the transit detection from the CoRoT light curves. Figure 3 

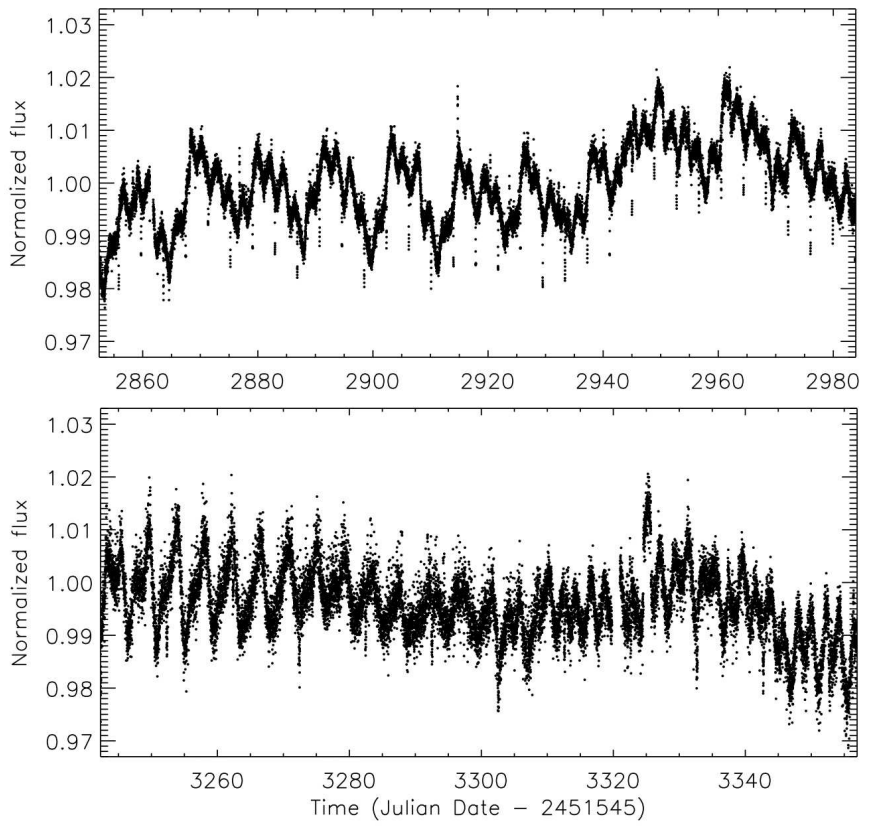

Figure 2: Two examples of CoRoT light curves .

shows the transit signal, defined here as the product of the relative depth of the transit and the square root of the number of points in the transit, as a function of the target magnitude. The color circles are the candidates identified, coded according to the run in which they were detected, and the black stars are the planets. This plot shows that Neptune size planets can be detected whatever the magnitude of the star is, whereas super-Earth size planets, such as CoRoT-7b can only be discovered in the light curves of stars brighter than $\mathrm{R} \simeq 14$.

\section{The CoRoT planets}

Table 2 gives the main characteristics of the CoRoT planets published by the end of summer 2010. These planets correspond to the first discoveries in the first two years of data only. Indeed, a large fraction of the candidates are still in the screening process by follow-up observations. Their properties, compared to the other published exoplanets are illustrated in Fig. 4. The mass-radius diagram shows that most of these planets are in the domain of gaseous giants but the long duration of the light curves and the high duty cycle (greater than 90\%) of space-based observations allow to explore the transiting systems over an extended orbital domain. The CoRoT discoveries indeed account for half of the transiting systems with orbital periods greater than 8 days.

\subsection{Extending the orbital domain of transiting planets}

The first discoveries, CoRoT-4b and $6 \mathrm{~b}$ are apart from the pile up of the period histogram below 5 days (Fig. 4). In the case of CoRoT-4b, the continuous photometry allows to measure the rotation period of the star and to establish that it is a synchro- 


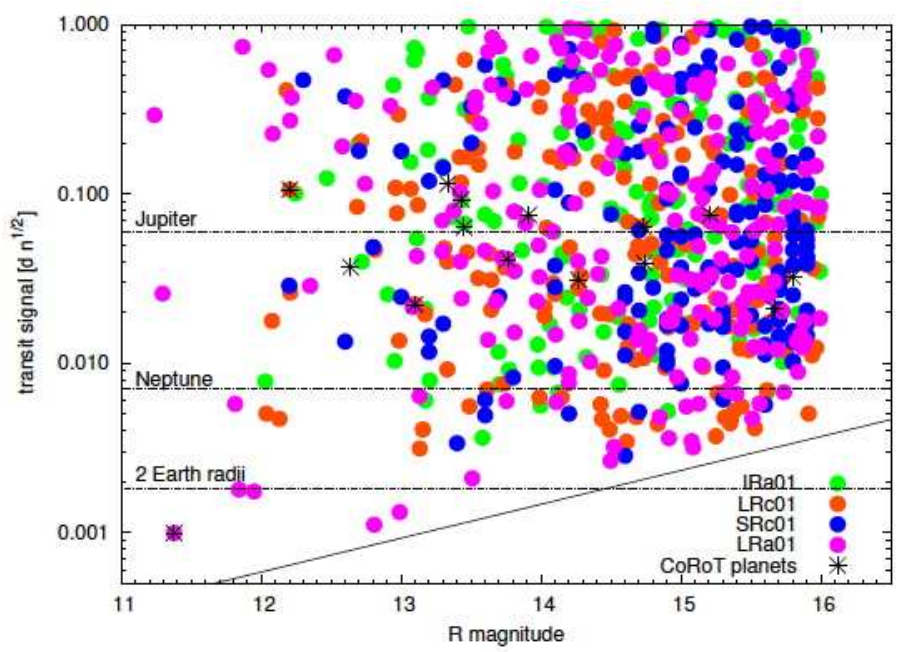

Figure 3: Transit signal for all candidates detected in the CoRoT/exoplanet fields observed during the first year (adapted from Cabrera et al. (2009)), with the expected signals for a Jupiter, Neptune and Earth-size like planets indicated.

nized system (Aigrain et al. 2008; Lanza et al. 2009). Among the CoRoT planets, it remains the only system for which the synchronization has been clearly detected. Not being synchronized despite a similar orbital period, CoRoT-6b constitutes a good case to probe for possible star-planet magnetic interactions. Lanza et al. (2010) used the light curve to map the longitudinal distribution of the photospheric active regions and trace their evolution. They reported some statistical evidence for active regions lagging the sub-planetary point during some temporal intervals. While the detection is still marginal, it is a good illustration of the interest of uninterrupted sequence of photometric observations to study the star - planet interaction and the evolution of planetary systems.

With an orbital period of 13 days, CoRoT-10b belongs to the class of the few transiting exoplanets with highly eccentric orbit (Bonomo et al. 2010). The large variation in the orbital distance along the planet's year, results in a tenfold increase in the amount of stellar radiation received by the planet. Its eccentricity could have been produced by Kozai oscillations with a distant companion of stellar nature or by planet - planet scattering. No hint of non-transiting companion has been reported so far but clearly this system would deserve radial velocity monitoring in order to further constrain the origin of the planet's eccentricity.

CoRoT-9b is the first long period transiting planet discovered by a transit survey (Deeg et al. 2010). The planet orbits its solar like G3-type star in 95 days. Unlike the two other long-period transiting planets, HD 17156b (Barbieri et al. 2007) and HD 80606b (Moutou et al. 2009), this Jupiter size planet has low eccentricity. This makes it a perfect representative of the known population of long-period planets (Fig. 5). CoRoT-9b is worth further deeper studies to probe the properties of this exoplanet population. 

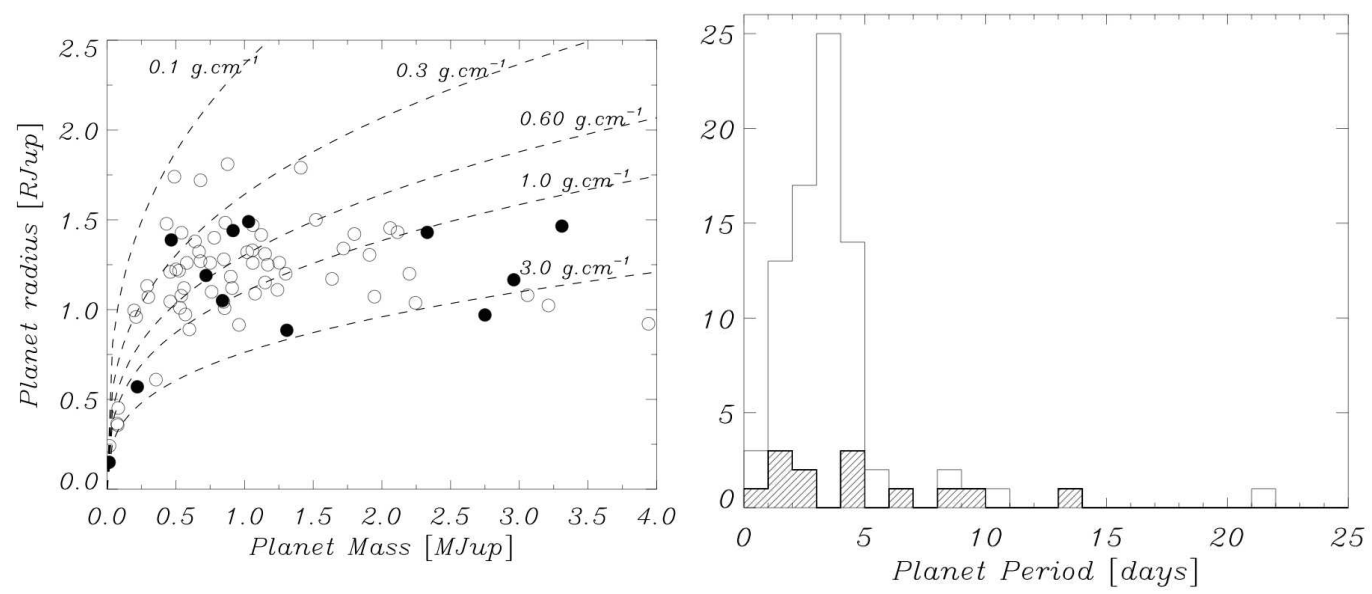

Figure 4: Mass - radius diagram for the transiting planets and orbital period distribution. Filled circles and the hashed histogram are the CoRoT planets.

\subsection{Populating the brown dwarf desert}

Exploring the exoplanet population at short orbital periods CoRoT has unexpectedly started to populate the so called brown-dwarf desert. While, with a mass of $21.6 \mathrm{M}_{J u p}$ for a Jupiters size, the exact nature of CoRoT-3b could be questioned as it lies in the overlapping region between the planet population and the low mass star regime (Deleuil et al. 2008), CoRoT-15b appears as a bona fide brown dwarf (Bouchy et al. 2010). Just as the most massive planets discovered so far, such as HAT-P-2b (Bakos et al. 2007) or CoRoT-14b (Tingley et al. 2010), these two massive companions orbit an F-type star with a marked vsini value. The significant rotational line broadening of the host star could be the cause of the lack of previous discoveries of such massive companions by radial velocity surveys, that usually either remove these objects from their sample after one measurement or set them aside with a very low observation ranking priority. This has resulted in a selection bias towards slow rotators that transit surveys have started to remove, allowing to enlarge the space of parameters for host stars. These discoveries reinforce the trend that close-orbiting companions to host stars with mass above $1.1 \mathrm{M}_{\odot}$ are more massive than companions to lower-mass hosts. Probing the physical properties of such objects could bring the missing clues to the understanding of the link between the population of planets and low-mass stars.

\subsection{Opening the small-size planet domain}

At the other extremity of the mass function, the recently discovered CoRoT-8b has a size in between Saturn and Neptune (Bordé et al. 2010) but a density comparable to that of Neptune. This suggests a massive core with a mass in the range 47 up to $64 \mathrm{M}_{\oplus}$ and a much smaller $\mathrm{H}$ - He envelope. Like HD 149026b, the parent star is metal rich, confirming the suspected trend for the formation of giant planets with high content of heavy elements for metal rich stars. In the mass - period diagram this planet lies in between the hot-Jupiter and the Super-Earth population, in a region where formation models predict a lack of planets. It might belong to the distribution tail of gaseous giants but clearly intensive exoplanet searches should be able to show 


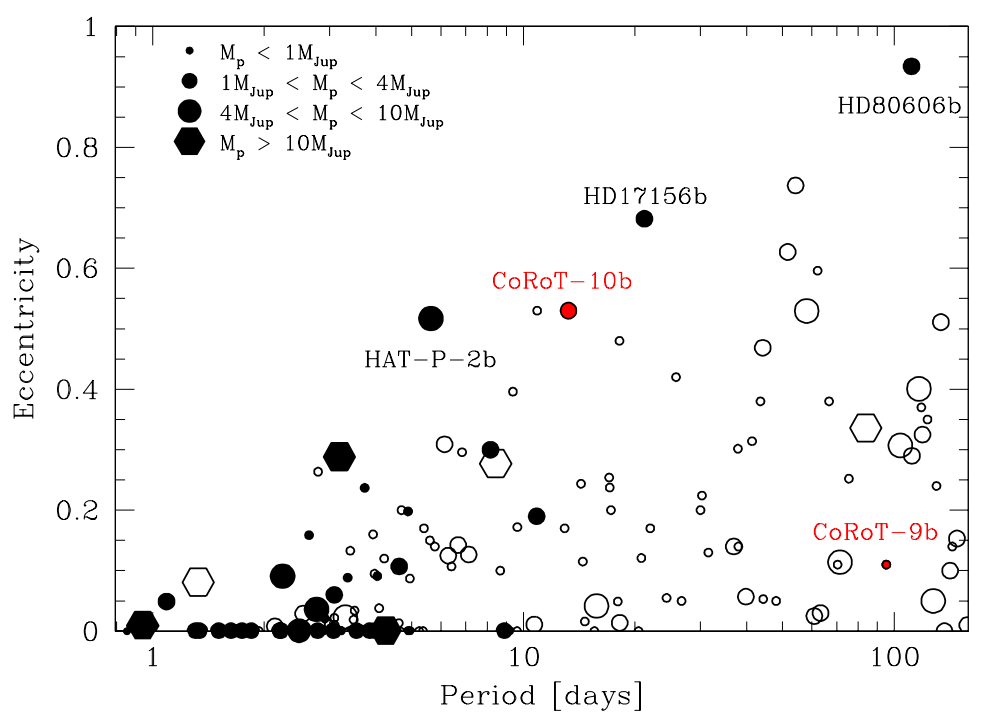

Figure 5: Eccentricity-period diagram for the known extrasolar planets (adapted from Bonomo et al. (2010)). Black filled symbols are transiting planets while open one are those with radial velocity measurements only. The size of the symbol indicates the mass range.

whether this region of the diagram remains as sparsely populated as predicted by a bimodal distribution.

Even smaller is CoRoT-7b, the first planet discovered in the domain of SuperEarth size planets. The planet orbits in 0.8 days a K0 main sequence star which is one of the brightest star in the first long run in the anti-center direction. The transit is $0.35 \mathrm{mmag}$ deep but is clearly detected in the Fourier transform of the light curve with all its harmonics. The star's light curve is modulated by spots at the surface of the star dragged by the star's rotation. This stellar activity produces a stellar jitter that competed the radial velocity measurements. More than 100 hundred HARPS spectra were collected over one year. Different methods have been used to analyze the radial velocity signal and to understand the effect of stellar activity (Queloz et al. 2009; Hatzes et al. 2010; Boisse et al. 2010; Pont et al. 2010). Whatever the study, they confirmed the planetary nature of CoRoT-7b with a mass that appears consistent with a rocky planet but with a poor precision, larger than $20 \%$. Not only the exact mass is subject to controversy but also the exact number of planets in the system. There are indeed some evidences in the radial velocity signal for a second non-transiting planet (Queloz et al. 2009) and even a third one (Hatzes et al. 2010). Clearly, to depict this system and accurately measure the mass of CoRoT-7b, additional velocity measurements are required with an adapted observation strategy so that the stellar activity imprint can be properly modelled.

\section{Conclusion}

For more than 3 years CoRoT has been collecting high precision photometry light curves. The first discoveries from the analysis of the first two years of data demonstrate 
the instrument capability to fully explore the domain of extra-solar planets at short orbital periods and to detect planets over a wide range of sizes and properties, from the first Super-Earth, CoRoT-7b, to CoRoT-9b the first temperate hot Jupiter. Continuous observations over long time spans also demonstrate their potential to probe different aspects of the planetary systems with, for example, the search for planet - host stars interactions. With a few tens of planet candidates in the follow-up process and new light curves to be analyzed and acquired in the two forthcoming years, new exciting results can be expected.

\section{References}

Aigrain, S., Collier-Cameron, A., Ollivier, M. et al. 2008, A\&A, 488, L43

Alonso, R., Auvergne, M., Baglin, A. et al. 2008, A\&A, 482, L21

Auvergne, M., Bodin, P., Boisnard, L. et al. 2009, A\&A, 506, 411

Baglin, A., Auvergne, M., Barge, P. et al. 2009, in IAU Symp. 253, Transiting Planets, 71 ed.

F. Pont et al.

Bakos, G., Kovács, G., Torres, G., et al. ApJ, 670, 826

Barbieri, M., Alonso, R., Laughlin, G. et al. 2007, A\&A, 476, 13

Barge, P., Baglin, A., Auvergne, M. et al. 2008, A\&A, 482, L17

Bonomo, A.S., Santerne, A., Alonso,R., et al. 2010, A\&A, 520, 65

Bordé, P., Bouchy, F., Deleuil, M. et al. 2010, A\&A, 520, 97

Bruntt, H., Deleuil, M., Fridlund, M., et al. 2010, A\&A, 519, 51

Boisse, I. et al. this volume

Bouchy, F., Moutou, C., Queloz, D. 2009 in IAU Symp. 253, Transiting Planets, 129 ed. F. Pont et al.

Bouchy, F., Deleuil, M., Guillot,T. et al. 2010, A\&A, 525, 68

Cabrera, J., Fridlund, M., Ollivier, M. et al. 2009, A\&A506, 501

Cabrera, J., Bruntt, H., Ollivier, M. et al. 2010, A\&A522, 110

Deeg, H. J., Gillon, M., Shporer, A., et al. 2009, A\&A, 506, 343

Deeg, H. J., Moutou, C., Erikson, A. et al. 2010, Nature, 464, 384

Deleuil, M., Deeg, H. J., Alonso, R. et al. 2008 A\&A, 491, 889

Fridlund, M., Hébrard, G., Alonso, R. et al. 2010, A\&A, 512, 14

Gandolfi, D., Hébrard, G.; Alonso, R. et al. 2010, A\&A, 524, 55

Gazzano, J.-C., de Laverny P., Deleuil M. et al. 2010, A\&A, 523, 91

Hatzes, A. P.; Dvorak, R., Wuchterl, G. et al. 2010, A\&A, 520, 93

Gillon, M., Lanotte, A. A., Barman, T. et al. 2010, A\&A, 511, 3

Lanza, A. F., Bonomo, A. S., Pagano, I. et al. 2010, A\&A, 525, 14L

Moutou, C., Hébrard, G., Bouchy, F. et al. 2009, A\&A, 498, 5

Pont, F., Aigrain, S. \& Zucker S. 2010, arXiv:1008.3859v2

Queloz,D., Bouchy,F., Moutou,C. et al. 2009, A\&A, 506, 303

Rauer, H., Queloz, D., Csizmadia, Sz. et al. 2009, A\&A, 506, 281

Surace, C., Alonso, R., Barge, P. et al. 2008, SPIE, 7019, 111

Tingley, B., Endl , M., Gazzano J.-C., et al., 2010, A\&A, in press 
Detection and Dynamics of Transiting Exoplanets

Table 2: Main characteristics of CoRoT discoveries.

\begin{tabular}{llcccccccc}
\hline Ref & Run & $\begin{array}{c}\text { Radius } \\
\mathrm{R}_{\text {Jup }}\end{array}$ & $\begin{array}{c}\text { Mass } \\
\mathrm{M}_{\text {Jup }}\end{array}$ & $\begin{array}{c}\text { Density } \\
\mathrm{gcm}^{-3}\end{array}$ & $\begin{array}{c}\text { Period } \\
\text { days }\end{array}$ & $\begin{array}{c}\mathrm{Nb} \\
\text { Type }\end{array}$ & $\begin{array}{c}\text { Star } \\
{[\mathrm{Fe} / \mathrm{H}]}\end{array}$ & Comment \\
\hline 1 & IRa01 & 1.49 & 1.03 & 0.34 & 1.509 & 34 & $\mathrm{G} 0 \mathrm{~V}$ & -0.20 & bloated HJ \\
2 & LRc01 & 1.46 & 3.31 & 1.31 & 1.743 & 78 & $\mathrm{G} 7 \mathrm{~V}$ & 0.00 & bloated HJ \\
3 & LRc01 & 1.01 & 21.6 & 26.4 & 4.26 & 34 & $\mathrm{~F} 3 \mathrm{~V}$ & -0.02 & BD or planet \\
4 & IRa01 & 1.19 & 0.72 & 0.52 & 9.20 & 7 & $\mathrm{~F} 8-9 \mathrm{~V}$ & +0.05 & synchronized \\
5 & LRa01 & 1.39 & 0.46 & 0.22 & 4.04 & 27 & $\mathrm{~F} 9 \mathrm{~V}$ & -0.25 & metal poor HS \\
6 & LRc02 & 1.16 & 2.96 & 2.32 & 8.89 & 15 & $\mathrm{~F} 9 \mathrm{~V}$ & -0.20 & metal poor HS \\
7 & LRa01 & 0.14 & $0.007-0.02$ & $3.4-9.6$ & 0.85 & 153 & $\mathrm{G} 9 \mathrm{~V}$ & 0.12 & Super-Earth \\
8 & LRc01 & 0.57 & 0.22 & 1.6 & 6.21 & 33 & $\mathrm{~K} 1 \mathrm{~V}$ & 0.30 & Icy giant \\
9 & LRc02 & 1.05 & 0.84 & 0.90 & 95.27 & 2 & $\mathrm{G} 3 \mathrm{~V}$ & -0.01 & temperate \\
10 & LRc01 & 0.97 & 2.75 & 3.70 & 13.24 & 10 & $\mathrm{~K} 1 \mathrm{~V}$ & 0.26 & eccentric \\
11 & LRc02 & 1.43 & 2.33 & 0.99 & 2.99 & 49 & $\mathrm{~F} 6 \mathrm{~V}$ & -0.03 & vsini $=40$ km/s \\
12 & LRa01 & 1.44 & 0.92 & 0.31 & 2.83 & 47 & $\mathrm{G} 4 \mathrm{~V}$ & 0.16 & bloated low density HJ \\
13 & LRa02 & 0.9 & 1.3 & 2.34 & 4.04 & 31 & $\mathrm{G0V}$ & 0.01 & dense HJ \\
14 & LRa02 & 1.09 & 7.6 & 7.3 & 1.15 & 89 & $\mathrm{~F} 9 \mathrm{~V}$ & 0.05 & extreme density \\
15 & SRa02 & 1.12 & 63.3 & 59 & 3.06 & 10 & $\mathrm{~F} 7 \mathrm{~V}$ & 0.10 & BD \\
\hline
\end{tabular}

The planets are ordered by increasing CoRoT number. HJ stands for Hot Jupiter and HS for host star. Column 7 gives the number of transits in the CoRoT light curve.(1) Barge et al. (2008); (2) Alonso et al (2008); (3) Deleuil et al (2008); (4) Aigrain et al (2008); (5) Rauer et al (2008); (6) Fridlund et al. (2010); (7) Léger et al. (2009); (8) Bordé et al. (2010); (9) Deeg et al. (2010); (10) Bonomo et al. (2010); (11) Gandolfi et al.(2010); (12) Gillon et al. (2010); (13) Cabrera et al. (2010); (14) Tingley et al. (2010); (15) Bouchy et al. (2010). 